die langfristig die Wettbewerbsfähigkeit verbessern.

Um die $\mathrm{CO}_{2}$-Emissionen bis zum Jahre $2005 \mathrm{im}$ angestrebten Umfang zu reduzieren, ist eine forcierte Politik zur Minderung des Energieverbrauchs durch eine rationellere Energienutzung und Energiebereitstellung sowie zur längerfristig-verstärkten Nutzung erneuerbarer Energiequellen überfällig. Die technischen Lösungen und deren wirtschaftliche Bewertung sind weitgehend bekannt. Worauf es heute ankommt, sind vor allem umfassende politische Maßnahmenpakete, die zur Nutzung der entsprechenden Potentiale führen. In den vergangenen Jahren wurden eine Reihe unterschiedlicher Maßnahmen zugunsten der rationellen und sparsamen Energieverwendung und der verstärkten Nutzung erneuerbarer Energiequellen geplant. Die wesentlichen Maßnahmen sind allerdings bisher nicht oder nur halbherzig umgesetzt worden.
Alles in allem lassen die bisherigen Aktivitäten eine Realisierung des selbstgesteckten Ziels als höchst unwahrscheinlich erscheinen.

Michael Kohlhaas, Barbara Praetorius Berlin

(1) Erklärung der deutschen Wirtschaft zur Klimavorsorge vom 10. März 1995, Bundesverband der Deutschen Industrie (BDI), Köln.

(2) Enquête-Kommission »Schutz der Erdatmosphäre « des Deutschen Bundestages (Hg.), Mehr Zukunft für die Erde. Nachhaltige Energiepolitik für dauerhaften Klimaschutz, Bonn 1995.

(3) Vgl. dazu Michael Kohlhaas und Barbara Praetorius, Selbstverpflichtungen der Industrie zur $\mathrm{CO}_{2}$-Reduktion, Sonderhefte des DIW Nr. 152, Berlin 1994.

\title{
Freiwillige Selbstverpflichtungen - Versuch einer Neubewertung
}

Die Spatzen pfeifen es von den Dächern: Das Instrument der freiwilligen Vereinbarungen ist derzeit angesagt in der Umweltpolitik. Die Bundesregierung präferiert offensichtlich dieses Instrumentarium der Umweltpolitik an Stelle ordnungsrechtlicher oder fiskalischer Instrumente (Ökosteuern), die Umweltverbände entdecken die Kooperation mit Unternehmen und ihren Verbänden als neues politisches Terrain und die Enquete-Kommission »Schutz des Menschen und der Umwelt « propagiert Kooperationen entlang der Wertschöpfungskette von Produkten unter dem neuen Begriff des Stoffstrommanagements. Cirka 5.000 freiwillige Vereinbarungen auf dem Gebiet der Umweltpolitik soll es in der Bundesrepublik geben.

Traditionell ist die Umweltökonomie sehr skeptisch gegenüber dem Instrumentarium der freiwilligen Vereinbarungen. Angesichts des Kollektivgutdilemmas gelten freiwillige Vereinbarungen i.d. R. allenfalls als second-best Lösungen, die hinsichtlich der ökonomischen Effizienz und vor allem hinsichtlich der ökologischen Lenkungswirkung weit hinter ordnungsrechtlichen und fiskalischen Instrumenten zurückstehen. Demnach muß gefragt werden, ob es Gründe gibt, diese Position zu revidieren. Werfen wir zu diesem Zweck zunächst einen Blick in die Praxis.

\section{Der Vorschlag der deutschen Wirtschaft}

Fast wie ein Beispiel aus dem Lehrbuch der Umweltökonomie mutet die Selbstverpflichtung der deutschen Wirtschaft zur Reduktion der $\mathrm{CO}_{2}$-Emissionen an: Am 10. März 95 wurde von den Verbänden der deutschen Wirtschaft eine Erklärung zur Klimavorsorge abgegeben. Sie setzt sich aus einer Sammelerklärung und Verpflichtungen der Einzelverbände zusammen. In der Erklärung zur Klimavorsorge heißt es:
»Auf freiwilliger Basis ist die deutsche Wirtschaft bereit, besondere Anstrengungen zu unternehmen, ihre spezifischen $\mathrm{CO}_{2}$-Emissionen bzw. den spezifischen Energieverbrauch bis zum Jahre 2005 (Basis 1987) um bis zu $20 \%$ zu verringern. «Im Gegenzug geht »... die Wirtschaft davon aus, daß die Bundesregierung auch zur Klimavorsorge (...) dieser Privatinitiative Vorrang vor ordnungsrechtlichen und fiskalischen Maßnahmen einräumt. «

Hier wird ein Tausch offeriert, der charakteristisch für viele freiwillige Vereinbarungen ist: Freiwillige Vereinbarungen dienen vielfach dazu, dem Staat den Verzicht auf drastischere umweltpolitische Maßnahmen abzuhandeln: in diesem Fall der Wärmenutzungsverordnung und bzw. oder eine $\mathrm{CO}_{2}$ /Energiesteuer. Prinzipiell ist gegen einen solchen Tausch auch nichts einzuwenden, vorausgesetzt, die freiwilligen Vereinbarungen leisten in etwa das gleiche was eine vergleichbare staatliche Maßnahme leisten würde. Hier sind erhebliche Zweifel angezeigt.

1. Das Żiel der Erklärung ist unklar. »... bis zu $20 \%$... k kann ebensogut auch nur $3 \%$ oder $5 \%$ Verringerung bedeuten. Ferner wird der Terminus »spezifisch« im Zusamenhang mit der Formulierung »Reduktion der spezifischen $\mathrm{CO}_{2}$-Emissionen « gemeinhin interpretiert als $\mathrm{CO}_{2}$-Emission pro Produkteinheit. Es ist also denkbar, daß eine Reduktion der $\mathrm{CO}_{2}$-Emissionen pro Produkteinheit um $20 \%$ realisiert wird und dennoch die $\mathrm{CO}_{2}$-Emissionen der Industrie, verursacht durch den absoluten Zuwachs an produzierten Produkten, steigen kann. Die $\mathrm{CO}_{2}$-Reduktionsziele, die die Bundesregierung selbst formuliert hat, $25 \%$ bis zum Jahre 2005, beziehen sich jedoch auf die absolute Menge der $\mathrm{CO}_{2}$-Emissionen und nicht auf die spezifischen.
(4) Vgl. Fraunhofer-Institut für Systemtechnik und Innovationsforschung, Potentiale und Kosten der Treibhausgasminderung im Industrieund Kleinverbrauchsbereich, Bericht für die Enquête-Kommission »Schutz der Erdatmosphäre« des Deutschen Bundestages, Studienkomplex B 3, Karsruhe 1994.

(5) Angaben für den Zeitraum von 1987 bis 2005 fallen noch höher aus, da von 1987 bis 1990 bereits ein deutlicher Rückgang des spezifischen Energieverbrauchs stattfand.

(6) Ziegelindustrie: nur alte Bundesländer; erwartete Entwicklung von 1987 bis 2005.

(7) Vgl. Stefan Bach, Michael Kohlhaas, Volker Meinhardt, Barbara Praetorius, Hans Wessels und Rudolf Zwiener, Wirtschaftliche Auswirkungen einer ökologischen Steuerreform, Sonderhefte des DIW Nr. 153, Berlin 1995.

2. Besondere Anstrengungen der Industrie scheinen zur Erfüllung dieser freiwilligen Erklärung nicht nötig zu sein. Interpretiert man die vorgegebenen $20 \%$ entgegen des Wortlautes der Erklärung als Absicht, die $\mathrm{CO}_{2}$-Emissionen der Industrie tatsächlich bis zum Jahre 2005 um $20 \% \mathrm{zu}$ verringern, dann stellt sich die Frage, welche Anstrengungen der Industrie abverlangt werden. Ein erhebliches Einsparungspotential ist der deutschen Industrie bereits durch den $\mathrm{Zu}$ sammenbruch der industriellen Basis in den neuen Bundesländern in den Schoß gefallen. $\mathrm{Da}$ die Energiekosten in Deutschland ohnehin im internationalen Maßstab vergleichsweise hoch sind, gibt es darüberhinaus in Deutschland jenseits aller freiwilligen Selbstverpflichtungen einen Trend in der Industrie zur Energieeinsparung durch technischen Fortschritt. Dadurch kommt es gleichsam automatisch zu einer Reduktion der $\mathrm{CO}_{2}$-Emissionen.

3. Der Erfolg dieser freiwilligen Selbstverpflichtung ist kaum nachvollziehbar. Der Blick in die Erklärung zeigt, daß Ziele zu Energie und $\mathrm{CO}_{2}$, spezifische und absolute Ziele sowie $\mathrm{Zie}$ le für neue und für alte Länder planlos nebeneinander stehen. Seitens des BDI wird denn auch zugegeben, daß die Vergleichbarkeit der Aussagen beschränkt ist. Die Erfahrungen des IÖW mit betrieblichen Ökobilanzen lassen darüber hinaus den klaren Schluß zu, daß die Aggregierung aller dieser Zahlen einzelner Unternehmen und ihrer Verbände in einem Datenfriedhof münden wird, aus dem sich ein Nachweis der Erfüllung oder Nichterfüllung der Ziele nie wird eindeutig ablesen lassen. Der BDI hätte durchsetzen müssen, $\mathrm{da} B$ in vergleichbaren Zahlen gerechnet wird. So z. B. entweder $\mathrm{CO}_{2}$ oder Energieverbrauch, entweder spezifisch oder absolut. Die je nach Verband unterschiedlichen Mittel des Monitoring runden dies Bild der Unkontrollierbarkeit ab.

4. Die freiwillige Erklärung des BDI führt zu einem Verzicht auf Instrumente zur $\mathrm{CO}_{2}$-Minde- 
rung, die andere Verursacher als die Industrie ebenso hätten treffen können. Denn neben der Industrie sind vor allem die privaten Haushalte und der Verkehr (mit steigender Tendenz) für die $\mathrm{CO}_{2}$-Emissionen verantwortlich.

Der ungebremste Anstieg des Energieverbrauchs im Verkehrssektors hat anteilig, genau wie der Sektor private Haushalte, den Industriebereich übertroffen und macht alle im industriellen Bereich erzielten Erfolge zunichte. Auch die mittlerweile von den Automobilherstellern (VDW) veröffentlichte Verpflichtung zur Reduktion des Kraftstoffverbrauches (19902005) um $25 \%$ wird durch das Mengenwachstum des Verkehrsaufkommens wieder aufgezehrt werden. Für diese Verursacher ist natürlich auch die Form einer freiwilligen Vereinbarung nicht geeignet. Kurz: Die Bundesregierung hat sich das Instrument einer Energiesteuer bzw. $\mathrm{CO}_{2}$-Abgabe von der Industrie abkaufen lassen und kann es jetzt auch dort, wo nie eine Vereinbarung wirken wird, nicht mehr einsetzen.

Im Ergebnis muß man die freiwillige Selbstverpflichtung des BDI als überwiegend symbolische Geste qualifizieren - Ausnahmen einzelner Industriezweige bestätigen die Regel -, mit der der Verzicht auf weitergehende staatliche Maßnahmen erreicht wwurde. Insofern entspricht diese freiwillige Selbstverpflichtung der Lehrbuchweisheit, wonach freiwillige Vereinbarungen bei Kollektivgütern entweder überhaupt nicht zustandekommen oder vielfach so vage ausfallen, daß sie einen Verzicht auf eine Veränderung der Rahmenbedingungen des wirtschaftlichen Handels durch den Staat kaum rechtfertigen können.

\section{Szenenwechsel: Veränderungen der Rahmenbedingungen - aber wie ?}

Man könnte das Thema freiwillige Vereinbarungen aus umweltökonomischer Sicht unter business as usual zu den Akten legen, wenn nicht ein kleiner ärgerlicher Umstand wäre: die Bereitschaft der Bundesregierung, sich auf diesen ungünstigen Tausch einzulassen, signalisiert, daß eine (ökologische) Veränderung der Rahmenbedingungen durch den Staat in einer demokratischen Gesellschaft auf Mehrheiten fußen muß und auf die Akzeptanz der wichtigsten sozialen Akteure angewiesen ist, zu denen zweifellos auch die Industrie zählt. In durchaus realistischer Einschätzung der politischen Verhältnisse geht die Bundesregierung offensichtlich davon aus, daß es an solchen Mehrheiten und an der Akzeptanz noch fehlt. In der Umweltökonomie fällt die Veränderung der Rahmenbedingungen gemeinhin wie Manna vom Himmel. In der umweltpolitischen Praxis ist das offensichtlich anders.

Die umweltökonomische Debatte krankt zutiefst daran, daß sie sich im wesentlichen auf den Vergleich der ökonomischen Effizienz und ökologischen Lenkungswirkung ausgewählter Instrumente kapriziert. Welche Motive die Akteure dazu veranlassen könnten, allokationsef- fiziente Instrumente zu implementieren bzw. ihre Implementation zu akzeptieren, ist ihr mehr und mehr aus dem Gesichtsfeld geraten. Auf die Frage »How the Patient follow the Doctor's Orders ?«, die Robert Hahn einem seiner Aufsätze als Titel gab, kann man immer noch getrost die Antwort geben: die Hunde bellen, die Karawane zieht weiter.

Offensichtlich muß sich die Blickrichtung ändern: Anstatt ständig die überlegene ökonomische Effizienz von marktwirtschaftlichen Instrumenten in der Umweltpolitik zu beschwören (woran ohnehin niemand ernstlich zweifelt), müssen sich gerade auch Ökonomen fragen, wie sich im Schoße einer modernen Gesellschaft überhaupt Verhältnisse entwickeln können, unter denen solche Instrumente wie etwa eine ökologische Steuerreform oder die Ausgabe von Umweltnutzungsrechten mehrheits- und vor allem seitens der wirtschaftlichen Akteure akzeptanzfähig werden. Um hier aus dem theoretisch toten Winkel herauszukommen, muß man sich von der gerade in der Volkswirtschaftslehre immer noch gängigen Vorstellung eines Unternehmens in Gestalt eines Reiz-Reaktions-Mechanismus lösen. Unternehmen tun mehr als nur veränderte Preis- und Mengensignale in theoretisch eindeutig ableitbare Handlungen umzusetzen. Sie machen Politik und können die Bedingungen ihres eigenen Handelns verändern. Bis zu einem gewissen Grade beeinflussen sie Art und Ausmaß des ökologischen Strukturwandels selbst.

Bis heute steht eine systematische theoretische Durchdringung dieser Handlungsmöglichkeiten aus. Dabei lassen sich auch mit theoretisch unbewaffnetem Auge ein ganze Reihe von Ansatzpunkten sogenannten proaktiven Verhaltens von Unternehmen erkennen. Dazu zählen

- informationelle Instrumente wie Umweltberichte, Produktbilanzen, Stoff- und Energiebilanzen und UVPs,

- Managementinstrumente wie das Ökocontrolling und Ökoaudits,

- neue institutionelle Arrangements wie unternehmensübergreifende Kooperationen im Rahmen eines Stoffstrommanagements und der Einbezug externe Anspruchsgruppen in Mediationsverfahren,

- Beeinflussung der Nachfrage durch LeastCost-Planning oder Ökoleasing,

und vieles andere mehr.

In welchem Ausmaß und in welchem Zeithorizont Unternehmen durch solche Instrumente zu einer »ökologischen Selbstveränderung « fähig sind, läßt sich gegenwärtig weder theoretisch noch empirišch eindeutig beantworten. Und die Beantwortung dieser Frage ebenso wie die Klärung des Problems, wie hier das Verhältnis von staatlichen Vorgaben und privater Initiative zu bestimmen ist, ist sicherlich des Schweißes der Edlen wert. Wie auch immer die Reichweite solcher Bemühungen beurteilt wird: Es macht einen gewichtigen Unterschied, ob auf der Bühne des politischen Bargainings um eine ökolo- gische Veränderung der Rahmenbedingungen wirtschaftlichen Handels mehr und mehr proaktive Unternehmen und ihre Verbände präsent sind oder nicht. Je mehr Akteure die Überzeugung gewinnen, daß ihre eigene Unternehmensund Verbandspolitik durch eine solche Veränderung faktisch belohnt wird, desto größer sind auch die Chancen für eine solche staatliche gesetzte Veränderung der Rahmenbedingungen. Denn faktisch verändern sich auf diese Weise auch die ökonomischen und politischen Incentives, die der Sicherung von Kollektivgütern wie der Umwelt im Wege stehen. Diese Veränderung muß nicht, kann aber zu einer Verbesserung der sozialen Voraussetzungen für die Überwindung des Kollektivgutdilemmas führen.

Noch einmal:

freiwillige Selbstverpflichtungen

Die allermeisten freiwilligen Selbstverpflichtungen bleiben weit hinter dem zurück, was aus umweltpolitischer Sicht wünschenswert wäre. Das ist kaum zu leugnen. Das ist aber auch nicht der geeignete Maßstab der Kritik. Interpretiert man sie jedoch als transitorisches Zwischenstadium zwischen völligem umweltpolitischem Attentismus und einer staatlich herbeigeführten Veränderung der (ökologischen) Rahmenbedingungen des Handelns, stehen sie für eine Zwischenetappe der gesellschaftlichen Selbstverständigung über Art und Ausmaß des erforderlichen ökologischen Strukturwandels. Sie. dokumentieren das Zugeständnis relevanter gesellschaftlicher Akteure, daß auf einem bestimmten Feld der Umweltpolitik Handlungsbedarf besteht und zugleich deren Absicht, bei der Befriedigung dieses Handlungsbedarfes möglichst billig - und das darf man vielfach wörtlich nehmen - davon zu kommen.

Vor diesem Hintergrund macht es auch nur einen begrenzten Sinn, freiwillige Selbstverpflichtungen alternativ zu anderen umweltpolitischen Instrumenten zu diskutieren. Es käme vielmehr darauf an, das politische Momentum, das.in aller Regel hinter der Bereitschaft zu einer freiwilligen Selbstverpflichtung steht, professionell zu nutzen. Exemplarisch: Wenn es kurzfristig schon nicht möglich ist, politisch eine Energiesteuer oder eine Wärmenutzungsverordnung umzusetzen, dann muß wenigstens die schwächere Selbstverpflichtung zur $\mathrm{CO}_{2}$ Emission eine nachprüfbare Substanz haben. Sie muß im Minimum

- ein unzweideutiges Mengenziel und Zeitziel haben,

- das Erreichen dieser Ziele muß im Rahmen eines geregelten Verfahrens meßbar und kontrollierbar sein,

- relevante Anspruchsgruppen wie die Umweltverbände sollten institutionalisierter Bestandteil des Bargaining-Prozesses wie des Monitoring sein,

- der Staat ebenso wie externe Anspruchsgruppen müssen während der Laufzeit einer solchen freiwilligen Selbstverpflichtung eine institutionalisierte exit-option haben, sofern 
absehbar wird, daß das Mengenziel in der fraglichen Zeit nicht erreicht wird,

- und schließlich sollte sich der Staat durch eine solche freiwillige Selbstverpflichtung einzelner Akteure nicht jene Handlungsmöglichkeiten abhandeln lassen, die andere Akteure tangieren.

Auf die Dauer kann auch eine freiwillige Selbstverpflichtung eine staatlich gesetzte Veränderung der Rahmenbedingungen nicht ersetzen. Diese könnte aber an Mehrheitsfähigkeit und Akzeptanz gewinnen, wenn unter dem Regime einer freiwilligen Selbstverpflichtung und anderer Kooperationsmechanismen ein gutes Stück des Weges zu einem ökologischen Strukturwandel der Wirtschaft zurückgelegt worden ist und dementsprechend die Anpassungskosten an einen über die Veränderung der Rahmenbedingungen induzierten ökologischen Strukturwandel sinken. Hannover, Berlin

\section{Neuerscheinung}

- DP 34/95 Christoph Kühleis: Ökologieverträgliche Zukunft der Arbeit?

Das Diskussionspapier beinhaltet einen Literatureinblick zur überwiegend sozialwissenschaftlich geführten Auseinandersetzung um eine ökologieverträgliche Zukunft der Arbeit. Der Beitrag skizziert zum einen die typischen Argumentationsmuster der aktuellen Diskussion und beschreibt die unterschiedlichen Ausgangspunkte, von denen sich die oft voneinander isolierten Debattenstränge dem Thema nähern. Insofern kann er auch zur thematischen Einführung dienen. Zum anderen zeigt er, die zum Teil noch sehr zarten Verbindungslinien zwischen der Kontroverse um die Zukunft der Erwerbsarbeitsgesellschaft und den Bestrebungen um eine Konkretisierung einer nachhaltigen Wirtschaftsweise auf. Damit versucht der Beitrag einen Zusammenhang zu umreißen, der bislang eher marginal und wenig systematisch behandelt wurde, dem gleichwohl beim ökologischen Umbau der Industriegesellschaft eine wichtige Bedeutung zukommt (vgl. auch den Beitrag von Rainer Lucas im letzten Informationsdienst 1/95, S. $5 / 6)$.

Ausgehend von der aktuellen Diskussion wird deren historische Entwicklung kurz nachgezeichnet. Anschließend wird die Rolle des informellen Sektors bzw. die Bedeutung der unbezahlten Arbeit in der Auseinandersetzung um die Zukunft der Arbeit behandelt. Hierauf folgt die Darstellung der Auseinandersetzung um die Zukunft der Erwerbsarbeit und ihrer ökologischen Anknüpfungspunkte. Darüber hinaus werden einige konkrete Reformvorschläge für den Weg zu einem ökologischen Wohlstandsmodell aufgegriffen.

\section{Instrumente des produktbezogenen Umwelt- schutzes - Das Beispiel Gerätebatterien}

\begin{abstract}
Das Heidelberger Regionalbüro des IÖW führt ein Projekt mit dem Titel »Produktpolitik zur Unterstïtzung der Umweltpolitik durch. Nach der Bestandsaufnahme produktpolitischer Instrumente in den Mitgliedstaaten der EU (vgl. Oosterhuis et. al. 1994) wurden im Rahmen dieser Studie, die von der EG Kommission finanziell unterstützt wird, ausgewählte Fallstudien erarbeitet (1). Neben den Instrumenten „Umweltzeichen und »öffentliches Beschaffungswesen wurden die auf die Produktgruppen "Farben und Lacke " und »Batterien « angewendeten Politiken näher untersucht. Die Fallbeispiele sind nach einem einheitlichen Muster strukturiert: Auf die Beschreibung der Umweltproblematik bzw. des Politikkontextes folgt eine detaillierte Evaluierung anhand bestimmter Kriterien. Anhand der Erfahrungen aus den Fallstudien werden in einer letzten Projektphase Optimierungsstrategien für den produktbezogenen Umweltschutz entwickelt. Das Projekt wird im Sommer 1995 abgeschlossen. Dieser Artikel widmet sich den Ergebnissen der Fallstudie »Gerätebatterien «.
\end{abstract}

Die wesentliche Umweltproblematik, die in der Regel mit Batterien verbunden wird, ist die Entsorgung der verbrauchten Zellen. Teilweise beträchtliche Anteile von Schwermetallen in den kleinen »Giftzwergen « - namentlich Quecksilber und Cadmium - machen die Deponierung bzw. Verbrennung zu einem aus ökologischer Sicht erheblichen Tatbestand (vgl. z. B. Baumann/Muth 1993). Die Problematik leitet sich aus der fast universellen Anwendbarkeit von Batterien aḅ. Allein in Deutschland werden jährlich an die 800 Millionen Batterien verkauft. Doch diese spezifische Umweltrelevanz, die vor allem die Entsorgungsphase des Produktlebens einer Batterie im Auge hat, vernachlässigt die allgemeine Umweltrelevanz, die sich am besten durch die Energiebilanz charakterisieren läßt: Der für die Herstellung einer Batterie nötige Energieinput übersteigt den Energieoutput über die gesamte Nutzungsdauer um das Fünfzigfache (Warmer Bulletin 35/92). Das verdeutlicht zum einen, daß eine systematischere Erfassung der Umweltrelevanz von Batterien im Sinne einer
Produktökobilanz angezeigt ist - dies ist bisher noch nicht geschehen. Zum anderen wird offensichtlich, daß Maßnahmen des produktbezogenen Umweltschutzes größere Optímierungspotentiale erschließen, wenn sie den gesamten Lebenszyklus einer Batterie mit in Betracht ziehen.

Wählen wir als Beispiel den Einsatz von Batterien in Uhren, so ist zunächst nach der Funktion zu fragen: Diese ist »Uhrzeit anzeigen«. Hieran kann gezeigt werden, daß nicht nur die Orientierung am Lebenszyklus ökologische Optimierungspotentiale offenlegt, sondern ebenso eine konsequentere Funktionsorientierung (Leinkauf/Zundel 1994). Die Uhrzeit kann von einer Sonnenuhr, einer mechanischen oder einer elektrischen Armbanduhr angezeigt werden (2). Die elektrische Armbanduhr kann wiederum per Solarzellen oder per Batterie betrieben werden. Wird die Batterie gewählt, so bleiben die Alternativen wiederaufladbare versus nicht-wiederaufladbare Batterie. Es ist offensichtlich, daß mit den je unterschiedlichen Problemlösungen unterschiedliche Umweltbelastungspotentiale verbunden sind und daß sich umweltpolitische Gestaltungsspielräume mit jeder Wahl einer bestimmten Problemlösung verringern.

\section{Umweltpolitik,}

Ziele und Instrumente

Gerätebatterien sind bereits seit einigen Jahren Gegenstand umweltpolitischer Maßnahmen, in Deutschland seit etwa Ende der siebziger Jahre. Das Abfallgesetz von 1986 gab der staatlichen Umweltpolitik die Möglichkeit an die Hand, für bestimmte Produkte und Substanzen Rücknahmepflichten einzuführen (Art. 14). Zwei Jahre später folgte die freiwillige Selbstverpflichtung von Batterieanbietern und Händlern, die gebrauchten Zellen freiwillig zurückzunehmen und für die umweltgerechte Entsorgung aufzukommen. Zudem verpflichtete sich die Herstellerseite, den Quecksilbergehalt insbesondere bei Alkali-Mangan Batterien beträchtlich zu reduzieren.

Nachdem die EG 1991 eine »Richtlinie über Batterien und Akkumulatoren, die gefährliche Substanzen enthalten «, verabschiedete, die neben Produkt- und Kennzeichnungsstandards die Sammlung und separate Entsorgung vorsieht, hat man sich in Deutschland auf Basis von Art. 14 des Abfallgesetzes um eine entsprechende Umsetzung bemüht. Der letzte veröffentlichte Verordnungsentwurf stammt aus dem Jahre 1992 und enthält eine gesetzliche Rücknahmepflicht für alle Batteriesysteme. Bis heute ist der Entwurf allerdings noch nicht verabschiedet. Aufgrund einer geänderten Rechtsbasis - 1996 tritt das neue Kreislaufwirtschaftsund Abfallgesetz in Kraft - ist die Umsetzungsfrist für die EG Richtlinie nun bereits um fast anderthalb Jahre überschritten. 
(c) 20I0 Authors; licensee IÖW and oekom verlag. This is an article distributed under the terms of the Creative Commons Attribution Non-Commercial No Derivates License (http://creativecommons.org/licenses/by-nc-nd/3.o/), which permits unrestricted use, distribution, and reproduction in any medium, provided the original work is properly cited. 